\title{
Einstein-Podolsky-Rosen correlations in second-harmonic generation
}

\author{
P. Lodahl \\ Department of Applied Physics and MESA + research institute, University of Twente, P.O. Box 217, 7500 AE Enschede, The Netherlands
}

(Received 12 February 2003; published 18 August 2003)

\begin{abstract}
A quantum model for singly resonant second-harmonic generation in a cavity with transverse degrees of freedom is analyzed. An instability threshold for pattern formation exists in this system. Below threshold, a strong modulation of the noise is demonstrated in the transverse structure of the far field. The performed analysis encompasses both one- and two-point correlation functions. The noise in a single far-field point is generally above the classical vacuum noise level. In contrast, strong nonclassical two-point correlations are encountered between opposite emission directions in the far field. For the fundamental field the correlations are shown to be of the Einstein-Podolsky-Rosen type, thus establishing a remarkable analog to the quantum correlations found in a nondegenerate optical parametric oscillator.
\end{abstract}

DOI: 10.1103/PhysRevA.68.023806

PACS number(s): 42.50.Dv, 03.65.Ud, 42.65.Sf

\section{INTRODUCTION}

The famous EPR paradox was put forward by Einstein, Podolsky, and Rosen in 1935 with the purpose of demonstrating the incompleteness of quantum mechanics [1]. By considering entangled states of two spatially separated quantum systems, EPR showed that a measurement on one subsystem would predict the outcome of a similar measurement on the other subsystem. This established quantum mechanics as a nonlocal theory, although this interpretation led EPR to reject the reality of the quantum description. Later developments of hidden variable theories were aiming at a restoration of the local realistic description of EPR correlations. The controversy was settled in the experiments by Aspect et al. [2], where a violation of the Bell inequality was realized, following a criteria by Bell for distinguishing local hidden variable theories from the quantum theory [3]. These experiments were utilizing discrete variables as, e.g., the polarization of single photons. In contrast, the original EPR proposal was for continuous variables, and the first experimental achievement of this type of correlations was by Kimble and co-workers based on the optical parametric oscillator (OPO) [4]. Recently, the nonlocality of continuous variable entanglement was also established experimentally [5]. Both of these experiments are based on continuous variables of light. Continuous variable quantum states of light have recently found applications as the quantum resource for fundamental quantum information processes, such as quantum teleportation [6,7] and dense coding [8], and have been proposed for use in continuous variable quantum cryptography [9].

The OPO has proven to be an efficient source for generating light with quantum correlations [10]. In the OPO, a nonlinear $\chi^{(2)}$ process converts the pump field at frequency $2 \omega$ into signal and idler fields $\omega_{\mathrm{s}}$ and $\omega_{\mathrm{i}}$. In the degenerate case $\left(\omega_{\mathrm{S}}=\omega_{\mathrm{i}}\right)$, squeezed light can be generated with noise reduction in one quadrature amplitude below the vacuum noise level $[11,12]$. In the nondegenerate optical parametric oscillator the signal and idler fields differ in polarization and/or frequency, which allows spatial separation of the two beams and the formation of continuous variable correlations of the type originally envisioned by EPR [13]. These correlations are observed as noise reduction below the vacuum level in the sum or difference of the signal and idler quadratures. In contrast, a single beam of the EPR pair exhibits excess fluctuations above the vacuum level. The presence of EPR correlations in the nondegenerate OPO can be attributed to the two-photon character of the parametric downconversion process that ensures strong correlations between signal and idler.

Spatiotemporal instabilities in cavity enhanced $\chi^{(2)}$ processes have attracted considerable attention. A large number of theoretical predictions [14-25] have recently been supplemented by experimental verifications of both a nonlinear self-pulsing instability [26] and first evidence for spatial instabilities $[27,28]$. Most work has concentrated on the OPO where off-axis emission of the parametric beams was predicted from a spatial instability $[14,15]$. This mechanism leads to the formation of modulated intensity patterns in the transverse plane perpendicular to the cavity axis [16]. Quantum spatial properties of the OPO have been studied intensively $[17,18]$ and spatial correlations and squeezing have been identified [19] as well as EPR correlations [20]. Second-harmonic generation ( $\mathrm{SHG}$ ) is the opposite process of the OPO. Here two fundamental photons are combined into a second-harmonic photon at twice the frequency: $\omega$ $+\omega \rightarrow 2 \omega$. The existence of an off-axis instability has also been established in SHG [21-23], enabling similar studies of spatial structures in SHG as in the OPO. Investigation of the quantum properties of this SHG instability was only initiated recently $[24,25]$, and the current work provides an extension of these studies.

It was recognized early that squeezed light correlations can be obtained not only in the OPO but also in SHG [29]. This is not surprising since the same nonlinear interaction $\left(\chi^{(2)}\right)$ is responsible for the two processes. In contrast, it does not seem immediately obvious that also EPR correlations can exist in SHG. The interacting fields in SHG are degenerate in frequency and polarization, which makes it not possible to separate two correlated beams. The SHG off-axis instability provides such a separation mechanism, allowing for the generation of spatially separated fields at both the fundamental and second harmonic frequencies. The main finding of the present paper is that these spatially separated fields can be EPR correlated. To the best of our knowledge, 


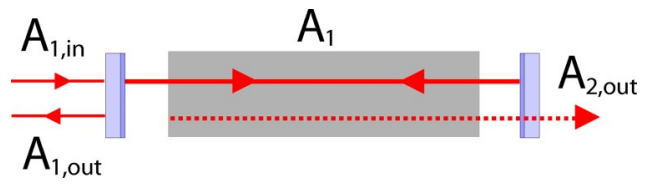

FIG. 1. (Color online) Sketch of the singly resonant SHG configuration. $A_{1}$ denotes the amplitude of the intracavity fundamental field while $A_{1 \text {,in/out }}$ are the input and output fundamental fields connected through the input coupling mirror. $A_{2, \text { out }}$ is the amplitude of the generated second harmonic field that freely escapes the cavity.

this is the first demonstration of nonlocal correlations in SHG. This ties a bond between spatially extended SHG and the nondegenerate OPO, where EPR correlations are also present. In addition, unique SHG correlations will be shown to exist that have no analogies in the OPO.

In the present paper we will study the spatial quantum properties of singly resonant SHG using the model first treated in Ref. [24]. After introducing the scaled quantized equations in Sec. II, the equations are solved in Sec. III in a linearized approximation valid below threshold for the spatial instability. In Secs. IV and V analytical expressions for one-point and two-point correlation functions are given, and the presence of both squeezing and EPR correlations simultaneously in the spatial structures is demonstrated.

\section{QUANTUM EQUATIONS FOR SINGLY RESONANT SHG}

In the most simple cavity enhanced frequency doubling configuration only the fundamental field $(\omega)$ is resonated, while the second harmonic $(2 \omega)$ escapes freely from the cavity. This is referred to as singly resonant SHG, cf. Fig. 1. A quantum model for this configuration was introduced by Collett and Levien [30] and extended by Paschotta et al. to squeezed light calculations [31]. The Collett-Levien model can be generalized to the spatially extended case, relevant for transverse quantum noise studies, by including a diffraction term [19]. As a result, the following operator equations are obtained:

$$
\begin{gathered}
\partial_{t} \hat{a}_{1}=\left(-\gamma_{1}+i \delta_{1}\right) \hat{a}_{1}+\chi^{2} f(\xi) \hat{a}_{1}^{\dagger} \hat{a}_{1}^{2}+\sqrt{2} i \chi g *(\xi) \hat{a}_{1}^{\dagger} \hat{a}_{2, \text { in }} \\
+i \frac{c^{2}}{2 \omega} \nabla_{\perp}^{2} \hat{a}_{1}+\sqrt{2 \gamma_{1}} \hat{a}_{1, \text { in }} \\
\hat{a}_{2, \text { out }}=\frac{i}{\sqrt{2}} \chi g(\xi) \hat{a}_{1}^{2}+\hat{a}_{2, \text { in }}
\end{gathered}
$$

Here $\hat{a}_{1}$ and $\hat{a}_{1}^{\dagger}$ are annihilation and creation operators for the intracavity fundamental field, $\hat{a}_{\mathrm{j} \text {,in }}, j=1,2$, account for input vacuum noise seeded to the fundamental and second harmonic fields, and the output quantum state of the second harmonic field is described by $\hat{a}_{2 \text {,out }}$. The equations contain linear and nonlinear terms. The former describe cavity loss and detuning with rates $\gamma_{1}$ and $\delta_{1}$. It is assumed that the input coupler transmission is the dominating loss for the fundamental. The diffraction term contains the speed of light in vacuum $c$ and the transverse Laplacian $\nabla_{\perp}^{2}=\partial^{2} / \partial x^{2}$ $+\partial^{2} / \partial y^{2}$ that contains the spatial degrees of freedom. The strength of the nonlinear terms is determined by the effective nonlinear coefficient $\chi$ and the phase mismatch parameter $\xi$. The phase mismatch turns out to be a convenient tuning parameter that contributes through the complex functions $f(\xi)=2 i / \xi+\left(e^{-2 i \xi}-1\right) / \xi^{2}$ and $g(\xi)=\left(e^{2 i \xi}-1\right) / \xi[22]$. We emphasize in Eq. (1a) the nonlinear coupling of fluctuations from the second harmonic field (through $\hat{a}_{2, \text { in }}$ ) into the intracavity fundamental field. This term only appears in a quantum model where vacuum fluctuations of the second harmonic input field are taken into account.

A proper description of the spatially extended system necessitates the use of a continuum of operators. The input noise operators obey the standard boson commutation relations

$$
\left[\hat{a}_{i, \text { in }}(\mathbf{r}, t), \hat{a}_{j, \text { in }}^{\dagger}\left(\mathbf{r}^{\prime}, t^{\prime}\right)\right]=\delta_{i j} \times \delta\left(\mathbf{r}-\mathbf{r}^{\prime}\right) \times \delta\left(t-t^{\prime}\right),
$$

where $\mathbf{r}=(x, y)$ is the transverse coordinate and $t$ is time. In general, these arguments will be omitted in the following for brevity. The photon number operator is defined as $\hat{n}_{\mathrm{i}, \mathrm{in}}$ $=\int d t \int d \mathbf{r} \hat{a}_{\mathrm{i}, \text { in }}^{\dagger}(\mathbf{r}, t) \hat{a}_{\mathrm{i}, \text { in }}(\mathbf{r}, t)$.

The output fundamental field, exiting through the same mirror that couples light into the cavity, is described by a standard input-output relation [32]

$$
\hat{a}_{1, \mathrm{out}}=\sqrt{2 \gamma_{1}} \hat{a}_{1}-\hat{a}_{1, \mathrm{in}} \text {. }
$$

Note that the intracavity operator $\hat{a}_{1}$ has been scaled such that the photon number operator inside the cavity is $\hat{n}_{1}$ $=1 / \tau \int d t \int d \mathbf{r} \hat{a}_{1}^{\dagger}(\mathbf{r}, t) \hat{a}_{1}(\mathbf{r}, t)$, where $\tau$ is the propagation time of one round trip in the cavity.

Equations (1a) and (1b) can be written in scaled form after the transformations $\sqrt{2 \omega \gamma_{1} / c^{2}} \mathbf{r} \rightarrow \mathbf{r}, \gamma_{1} t \rightarrow t$, and defining $\quad \hat{A}_{1}=\chi \hat{a}_{1} / \sqrt{\gamma_{1}}, \quad \hat{A}_{1 \text {,in }}=\sqrt{2} \chi \hat{a}_{1 \text {,in }} / \gamma_{1}, \quad \hat{A}_{2 \text {,in/out }}$ $=\sqrt{2} \chi \hat{a}_{2, \text { in out }} / \gamma_{1}, \Delta_{1}=\delta_{1} / \gamma_{1}$. All scaled parameters are unitless. We arrive at the equations

$$
\begin{gathered}
\partial_{t} \hat{A}_{1}=\left(-1+i \Delta_{1}\right) \hat{A}_{1}+f(\xi) \hat{A}_{1}^{\dagger} \hat{A}_{1}^{2}+i g *(\xi) \hat{A}_{1}^{\dagger} \hat{A}_{2, \text { in }} \\
+i \nabla_{\perp}^{2} \hat{A}_{1}+\hat{A}_{1, \text { in }}, \\
\hat{A}_{2, \text { out }}=i g(\xi) \hat{A}_{1}^{2}+\hat{A}_{2, \text { in }} .
\end{gathered}
$$

The commutation relations of the scaled amplitudes are given by

$$
\left[\hat{A}_{\mathrm{j}, \text { in }}(\mathbf{r}, t), \hat{A}_{\mathrm{j}, \text { in }}^{\dagger}\left(\mathbf{r}^{\prime}, t^{\prime}\right)\right]=\kappa \delta\left(\mathbf{r}-\mathbf{r}^{\prime}\right) \times \delta\left(t-t^{\prime}\right),
$$

with $j=1,2$ and $\kappa=4 \chi^{2} \omega / c^{2}$. The input-output relation of Eq. (3) transforms into

$$
\hat{A}_{1, \mathrm{out}}=2 \hat{A}_{1}-\hat{A}_{1, \text { in }},
$$

with $\hat{A}_{1, \text { out }}=\sqrt{2} \chi \hat{a}_{1, \text { out }} / \gamma_{1}$.

Equations (4) are the scaled quantized equations for singly resonant SHG that will be examined in the current work. The classical versions of these equations were studied in de- 
tail in Refs. [22,23]. Here it was shown that a spatial instability exists for certain values of cavity detuning $\Delta_{1}$ and phase mismatch $\xi$. Above the instability threshold, spatially modulated patterns can be excited as, e.g., squares or hexagons. The mechanism for pattern formation was found to be off-axis emission characterized by a critical transverse wave vector,

$$
k_{\mathrm{c}}=\sqrt{\Delta_{1}+\Delta_{1}^{\mathrm{NL}}},
$$

with the nonlinear detuning $\Delta_{1}^{\mathrm{NL}}=2 f_{\mathrm{i}}(\xi)\left|A_{1}\right|^{2}$, and subscript i denotes the imaginary part. This establishes the mechanism for pattern formation in SHG as being similar to the OPO case, i.e., compensation of cavity detuning by off-axis emission [14].

Here we concentrate on the quantum properties. It will be shown that below threshold for the spatial instability, the fluctuations are spatially modulated and possess correlations beyond the classical limit. Such structures have coined the name quantum images [33]. In contrast, the corresponding averaged amplitudes $A_{1, \text { out }}$ and $A_{2, \text { out }}$ are homogeneous solutions with no spatial modulation.

\section{ANALYTICAL SOLUTIONS OF LINEARIZED EQUATIONS}

The nonlinear quantum model of singly resonant SHG in Eq. (4) is solved within the framework of the semiclassical approximation [34]. With this technique the operator equations are linearized, allowing for analytical solutions. We substitute $\hat{A} \rightarrow A+\hat{b}$, where $A$ is the averaged amplitude of each field and the operator $\hat{b}$ accounts for the quantum fluctuations. The assumption behind this approximation is that the quantum noise is a small perturbation to the classical amplitudes. In that case Eq. (4) can be linearized in $\hat{b}$, leading to

$$
\begin{gathered}
\partial_{t} \hat{b}_{1}=\left(-1+i \Delta_{1}\right) \hat{b}_{1}+2 f(\xi)\left|A_{1}\right|^{2} \hat{b}_{1}+f(\xi) A_{1}^{2} \hat{b}_{1}^{\dagger}, \\
+i g^{*}(\xi) A_{1}^{*} \hat{b}_{2, \text { in }}+i \nabla_{\perp}^{2} \hat{b}_{1}+\hat{b}_{1, \text { in }}, \\
\hat{b}_{2, \text { out }}=2 i g(\xi) A_{1} \hat{b}_{1}+\hat{b}_{2, \text { in }},
\end{gathered}
$$

which can be solved analytically relating the output fields to the input fields. The semiclassical equations can be directly used to calculate expectation values of symmetrically ordered operator products, thus applicable to the quadrature correlations treated below. The method has proven its impressive validity for quantum noise calculations of $\chi^{(2)}$ nonlinear systems, and is expected to be of high validity for SHG where a large averaged amplitude is always present for all pump levels. For a thorough discussion of the semiclassical approximation, see Ref. [34].

The averaged amplitude of the fundamental field inside the cavity, $A_{1}$, is found by solving the classical equation [22]. Quantum noise coupled into the cavity is expressed by the operators $\hat{b}_{1 \text {,in }}$ and $\hat{b}_{2 \text {,in }}$. The noise in the fundamental field is introduced by the pump field which is assumed to be in a coherent state. For the second harmonic field no pump is applied, i.e., the input is vacuum noise. At optical frequencies thermal photons can be safely neglected, and the following correlation relations for the input operators hold [35]:

$$
\begin{gathered}
\left\langle\hat{b}_{\mathrm{j}, \mathrm{in}}(\mathbf{r}, t)\right\rangle=\left\langle\hat{b}_{\mathrm{j}, \mathrm{in}}^{\dagger}(\mathbf{r}, t)\right\rangle=0, \\
\left\langle\hat{b}_{\mathrm{j}, \mathrm{in}}^{\dagger}(\mathbf{r}, t) \hat{b}_{\mathrm{j}, \mathrm{in}}\left(\mathbf{r}^{\prime}, t^{\prime}\right)\right\rangle=0, \\
\left\langle\hat{b}_{\mathrm{j}, \mathrm{in}}(\mathbf{r}, t) \hat{b}_{\mathrm{j}, \mathrm{in}}^{\dagger}\left(\mathbf{r}^{\prime}, t^{\prime}\right)\right\rangle=\kappa \delta\left(\mathbf{r}-\mathbf{r}^{\prime}\right) \times \delta\left(t-t^{\prime}\right),
\end{gathered}
$$

in accordance with the commutation relations in Eq. (5).

The linear differential equation (8a) is most easily solved after a spatiotemporal Fourier transformation $\hat{\beta}_{j}(\Omega, \mathbf{k})$ $=\int d \mathbf{r} \int d t \hat{b}_{j}(\mathbf{r}, t) e^{i(\mathbf{k} \cdot \mathbf{r}+\Omega t)}, j=1,2$. The operators $\hat{\beta}_{j}(\mathbf{k}, \Omega)$ account for quantum noise at a frequency $\Omega$ at a transverse wave vector $\mathbf{k}$ in the far field. The Fourier transformed equation is

$$
\begin{aligned}
c_{1}\left(\Omega, k^{2}\right) \hat{\beta}_{1}(\Omega, \mathbf{k})= & c_{2} \hat{\beta}_{1}^{\dagger}(-\Omega,-\mathbf{k})+c_{3} \hat{\beta}_{2, \text { in }}(\Omega, \mathbf{k}) \\
& +\hat{\beta}_{1, \text { in }}(\Omega, \mathbf{k}),
\end{aligned}
$$

where the following coefficients have been introduced:

$$
\begin{gathered}
c_{1}\left(\Omega, k^{2}\right)=1-2 f(\xi)\left|A_{1}\right|^{2}-i\left(\Omega+\Delta_{1}-k^{2}\right), \\
c_{2}=f(\xi) A_{1}^{2}, \\
c_{3}=i g *(\xi) A_{1}^{*} .
\end{gathered}
$$

This equation is solved by applying the input-output relations (4b) and (6). This leads to

$$
\begin{aligned}
\hat{\beta}_{1, \text { out }}(\Omega, \mathbf{k})= & \left(\bar{c}_{1}-1\right) \hat{\beta}_{1, \text { in }}(\Omega, \mathbf{k})+\bar{c}_{2} \hat{\beta}_{1, \text { in }}^{\dagger}(-\Omega,-\mathbf{k}) \\
& +\bar{c}_{3} \hat{\beta}_{2, \text { in }}(\Omega, \mathbf{k})+\bar{c}_{4} \hat{\beta}_{2, \text { in }}^{\dagger}(-\Omega,-\mathbf{k}),
\end{aligned}
$$

$\hat{\beta}_{2, \text { out }}(\Omega, \mathbf{k})=i g(\xi) A_{1} \bar{c}_{1} \hat{\beta}_{1, \text { in }}(\Omega, \mathbf{k})+i g(\xi) A_{1} \bar{c}_{2}$

$$
\begin{aligned}
& \times \hat{\beta}_{1, \text { in }}^{\dagger}(-\Omega,-\mathbf{k})+\left[1+i g(\xi) A_{1} \bar{c}_{3}\right] \hat{\beta}_{2, \text { in }}(\Omega, \mathbf{k}) \\
& +i g(\xi) A_{1} \bar{c}_{4} \hat{\beta}_{2, \text { in }}^{\dagger}(-\Omega,-\mathbf{k}),
\end{aligned}
$$

with the frequency and wave vector dependent coefficients given by

$$
\begin{aligned}
& \bar{c}_{1}\left(\Omega, k^{2}\right)=\frac{2 c_{1}^{*}\left(\Omega, k^{2}\right)}{\left|c_{1}\left(\Omega, k^{2}\right)\right|^{2}-\left|c_{2}\right|^{2}}, \\
& \bar{c}_{2}\left(\Omega, k^{2}\right)=\frac{2 c_{2}}{\left|c_{1}\left(\Omega, k^{2}\right)\right|^{2}-\left|c_{2}\right|^{2}}, \\
& \bar{c}_{3}\left(\Omega, k^{2}\right)=\frac{2 c_{1}^{*}\left(\Omega, k^{2}\right) c_{3}}{\left|c_{1}\left(\Omega, k^{2}\right)\right|^{2}-\left|c_{2}\right|^{2}}, \\
& \bar{c}_{4}\left(\Omega, k^{2}\right)=\frac{2 c_{2} c_{3}^{*}}{\left|c_{1}\left(\Omega, k^{2}\right)\right|^{2}-\left|c_{2}\right|^{2}} .
\end{aligned}
$$


These rather extensive expressions are the solutions relating the output electric field operators for the fundamental and second harmonic to the input fields. $\chi^{(2)}$ quantum correlations are most conveniently expressed in quadrature amplitudes. Focusing only on far-field correlations, we define the quadratures

$$
\hat{P}_{\mathrm{j}, \text { in } / \text { out }}(\theta, \Omega, \mathbf{k})=\hat{\beta}_{\mathrm{j}, \text { in } / \text { out }}(\Omega, \mathbf{k}) e^{-i \theta}+\hat{\beta}_{\mathrm{j}, \text { in } / \text { out }}(-\Omega, \mathbf{k}) e^{i \theta},
$$

characterized by the quadrature phase angle $\theta$. Quadrature amplitudes are naturally measured in balanced homodyne detection [36]. From Eq. (9) it is easy to show that

$$
\left\langle\hat{P}_{1, \text { in }}(\theta, \Omega, \mathbf{k}) \hat{P}_{1, \text { in }}\left(\theta, \Omega^{\prime}, \mathbf{k}^{\prime}\right)\right\rangle=\kappa \delta\left(\mathbf{k}-\mathbf{k}^{\prime}\right) \delta\left(\Omega+\Omega^{\prime}\right),
$$

$$
\left\langle\hat{P}_{2, \text { in }}(\theta, \Omega, \mathbf{k}) \hat{P}_{2, \text { in }}\left(\theta, \Omega^{\prime}, \mathbf{k}^{\prime}\right)\right\rangle=\kappa \delta\left(\mathbf{k}-\mathbf{k}^{\prime}\right) \delta\left(\Omega+\Omega^{\prime}\right) .
$$

These input correlation functions provide the proper normalization for the output correlation functions discussed in the following sections. It will be shown that the output quadrature correlations can be reduced below the input, which is a nonclassical phenomenon.

\section{ONE-POINT CORRELATIONS}

In this section we concentrate on one-point temporal correlations by calculating the quantum noise in a single spatial far-field point $\mathbf{k}$. The quadrature noise spectrum is defined as

$$
S_{\mathrm{j}}(\theta, \Omega, \mathbf{k})=\frac{\left\langle\hat{P}_{\mathrm{j}, \text { out }}(\theta, \Omega, \mathbf{k}) \hat{P}_{\mathrm{j}, \text { out }}(\theta,-\Omega, \mathbf{k})\right\rangle}{\left\langle\hat{P}_{\mathrm{j}, \text { in }}(\theta, \Omega, \mathbf{k}) \hat{P}_{\mathrm{j}, \mathrm{in}}(\theta,-\Omega, \mathbf{k})\right\rangle},
$$

which measures the output quadrature noise relative to the vacuum noise level. Squeezed light corresponds to $S_{\mathrm{j}} \leqslant 1$, i.e., noise reduction below the vacuum noise level. Based on the solutions in Eqs. (12), the noise spectrum is given,

$$
\begin{aligned}
S_{\mathrm{j}}(\theta, \Omega, \mathbf{k})= & u_{\mathrm{j}}\left(\Omega, k^{2}\right)+\left[v_{\mathrm{j}}\left(\Omega, k^{2}\right) e^{-2 i \theta}\right. \\
& \left.+v_{\mathrm{j}}^{*}\left(\Omega, k^{2}\right) e^{2 i \theta}\right] \frac{\delta(2 \mathbf{k})}{\delta(\mathbf{0})},
\end{aligned}
$$

where the following functions have been defined:

$$
\begin{gathered}
u_{1}\left(\Omega, k^{2}\right)=\left|\bar{c}_{1}\left(\Omega, k^{2}\right)-1\right|^{2}+\left|\bar{c}_{2}\left(\Omega, k^{2}\right)\right|^{2}+\left|\bar{c}_{3}\left(\Omega, k^{2}\right)\right|^{2} \\
+\left|\bar{c}_{4}\left(\Omega, k^{2}\right)\right|^{2}, \\
v_{1}\left(\Omega, k^{2}\right)=\left[\bar{c}_{1}\left(\Omega, k^{2}\right)-1\right] \bar{c}_{2}\left(\Omega, k^{2}\right)+\bar{c}_{3}\left(\Omega, k^{2}\right) \bar{c}_{4}\left(\Omega, k^{2}\right),
\end{gathered}
$$

$$
\begin{aligned}
u_{2}\left(\Omega, k^{2}\right)= & \left|g(\xi) A_{1}\right|^{2}\left[\left|\bar{c}_{1}\left(\Omega, k^{2}\right)\right|^{2}+\left|\bar{c}_{2}\left(\Omega, k^{2}\right)\right|^{2}\right] \\
& +\left|1+i g(\xi) A_{1} \bar{c}_{3}\left(\Omega, k^{2}\right)\right|^{2} \\
& +\left|g(\xi) A_{1}\right|^{2}\left|\bar{c}_{4}\left(\Omega, k^{2}\right)\right|^{2}
\end{aligned}
$$

$$
\begin{aligned}
v_{2}\left(\Omega, k^{2}\right)= & -g^{2}(\xi) A_{1}^{2} \bar{c}_{1}\left(\Omega, k^{2}\right) \bar{c}_{2}\left(\Omega, k^{2}\right)+i g(\xi) A_{1} \\
& \times\left[1+i g(\xi) A_{1} \bar{c}_{3}\left(\Omega, k^{2}\right)\right] \bar{c}_{4}\left(\Omega, k^{2}\right) .
\end{aligned}
$$

Due to the Dirac $\delta$ functions in Eq. (17), a natural division arises between $k=0$ and $k \neq 0$. The first case corresponds to the situation where diffraction is excluded. In that limit the squeezing calculations of previous works can be reproduced [31]. However, the model presented here is more general and includes both cavity detuning and phase mismatch of the nonlinear process. In far-field points where $k \neq 0$, the observed light has been subjected to diffraction, which implies a spatially modulated noise distribution. The sharp transition between these two regions, as expressed by the $\delta$ function, is due to the assumption of an infinitely extended plane wave pump field, which corresponds to a single point $(k=0)$ in the far field.

As discussed above, squeezing can occur in the part of the far-field image that has not been affected by diffraction. Inserting $k=0$ in Eq. (17) leads to

$$
S_{\mathrm{j}}(\theta, \Omega, 0)=u_{\mathrm{j}}(\Omega, 0)+v_{\mathrm{j}}(\Omega, 0) e^{-2 i \theta}+v_{\mathrm{j}}^{*}(\Omega, 0) e^{2 i \theta} .
$$

Here the noise spectrum is seen to depend on the quadrature angle $\theta$ as is characteristic for phase sensitive nonlinear processes, of which SHG is an example. This enables noise reduction of one quadrature $\hat{P}(\theta)$ below the vacuum noise level (squeezing) at the cost of increased noise in $\hat{P}(\theta$ $+\pi / 2)$. The phase mismatch parameter $\xi$ turns out to be an important tuning parameter for the optimization of squeezing. Figure 2 shows squeezing spectra of the fundamental and second harmonic fields at resonance $\left(\Delta_{1}=0\right)$, and for three different values of the phase mismatch. For perfect phase matching $(\xi=0)$, best squeezing for the fundamental field is seen to be at zero frequency. Quite remarkably, substantially better squeezing performance is predicted for nonzero phase mismatch. Indeed, for $\xi= \pm \pi$ noise reduction to better than 0.4 times the vacuum noise level is obtained exceeding the maximum squeezing level of $2 / 3$ for phasematched singly resonant SHG [31]. Furthermore, for $\xi<0$ the strongest noise reduction can be obtained at a nonzero frequency. This will be advantageous in an experiment since technical noise is more pronounced at low frequencies $(\Omega$ $\rightarrow 0$ ). In the second harmonic [Fig. 2(b)] best squeezing is found at zero frequency and for zero phase mismatch.

For far-field points with $\mathbf{k} \neq 0$, it follows from Eq. (17) that

$$
S_{\mathrm{j}}(\theta, \Omega, \mathbf{k} \neq 0,)=u_{\mathrm{j}}\left(\Omega, k^{2}\right) .
$$

In this case the noise spectrum is found to be independent of the quadrature angle $\theta$ and is always above the vacuum noise level, i.e., $S_{\mathrm{j}}(\theta, \Omega, k \neq 0) \geqslant 1$. In contrast to the light emitted with $k=0$ (the homogeneous solution), the off-axis emitted fields are not squeezed and contain substantial excess noise. For cavity and pump parameters, where a spatial instability exists in the system, the noise spectrum is found to be strongly modulated. An example is shown in Fig. 3 for a 

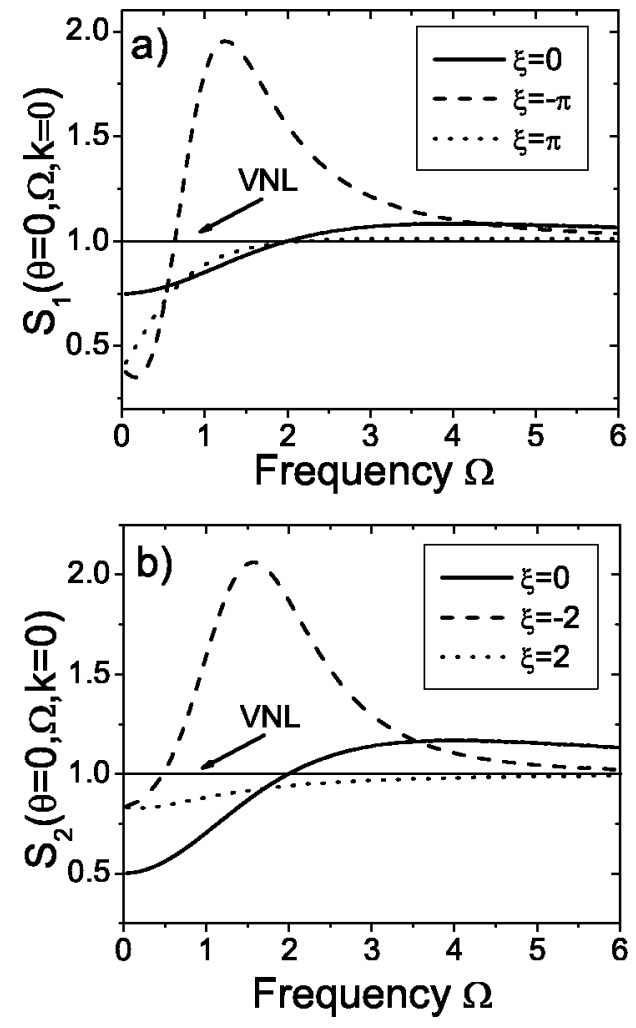

FIG. 2. Fundamental and second harmonic squeezing spectra as a function of frequency for different values of the phase mismatch parameter $\xi$. The fundamental detuning has been fixed at resonance $\Delta_{1}=0$, the quadrature phase angle is $\theta=0$, and the intracavity scaled intensity is $\left|A_{1}\right|^{2}=0.5$. Squeezing is noise reduction below the vacuum noise level (VNL), which is marked with a horizontal line in the plots.

pump level below threshold for the spatial instability. The noise is clearly structured and forms a modulation ring that increases in amplitude as approaching threshold. Similarly the radius of the ring approaches the critical instability wave number $k_{\mathrm{c}}$. This excess noise is due to spontaneous emission of photons in the off-axis beams that are present also below

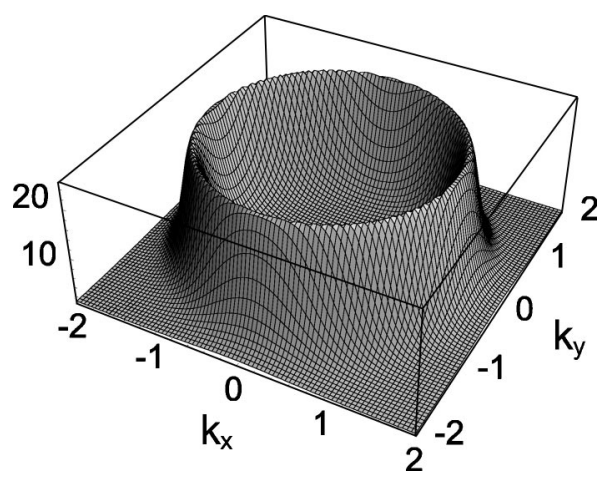

FIG. 3. Fundamental noise spectrum $S_{1}(\theta=0, \Omega=0, \mathbf{k})$ plotted in the far-field transverse plane $\left(k_{x}, k_{y}\right)$ for $\Delta_{1}=0$ and $\xi=5$. The cavity pump level is chosen such that the scaled intracavity intensity is $\left|A_{1}\right|^{2}=2.5$, which is below threshold for the spatial instability that sets in at $\left|A_{1}\right|^{2}=3.56$ with a critical wave number $k_{\mathrm{c}}$ $=1.73$.

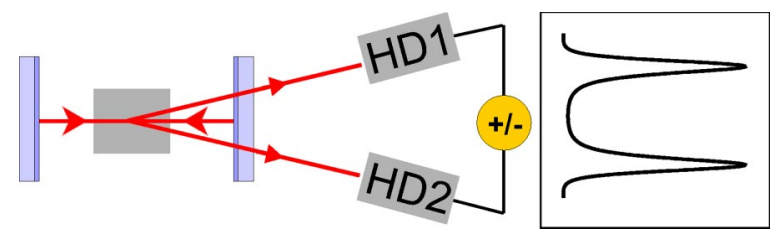

FIG. 4. (Color online) Sketch illustrating the technique of measuring spatial two-point correlation functions. Two homodyne detectors (HD1 and HD2) record quadrature noise at spatially separated points in the far field. Each homodyne detector will record excess noise above the vacuum noise level as indicated by the inset spectrum that represents a cut through the emission ring of Fig. 3. The quantum correlations are revealed by adding or subtracting the photocurrents from the two homodyne detectors.

instability threshold. This behavior has strong analogies to what is found in a nondegenerate OPO below oscillation threshold [13]. In that case the quantum noise of both the signal and idler beams is quadrature independent and above the vacuum noise level. In fact, the similarities between the off-axis emitted beams in SHG and the signal and idler beams of the OPO hold even further. As will be shown in the following section, the emission in two opposite points on the modulation ring turns out to be strongly correlated beyond the vacuum noise limit. In fact, these correlations will be shown to be of the EPR type, similar to what can be found in the nondegenerate OPO. This remarkable link between spatially separated SHG and the OPO can be given some intuitive backup from the fact that nonzero phase mismatch ensures that not only the SHG conversion $\omega+\omega \rightarrow 2 \omega$ occurs in the nonlinear process but also the opposite downconversion $2 \omega \rightarrow \omega+\omega$. The instability provides spatial separation of two beams, allowing for two-point correlation studies. The many quantitative similarities between the nondegenerate OPO and spatially extended SHG are striking. There also appear significant differences, the most pronounced being that in SHG also nonclassical two-point correlations are found in the second-harmonic field. This has no counterpart in the nondegenerate OPO where the separation of two harmonic beams is not possible.

\section{EPR CORRELATIONS}

While the noise in spatial points $k \neq 0$ was found to be above the vacuum noise level and hence classical, the quantum behavior is revealed when considering two-point correlation functions for two opposite points on the emission ring. These two-point correlations can be measured by adding or subtracting the photocurrents from two homodyne detectors probing the wave vectors $\mathbf{k}$ and $\mathbf{- k}$ in the far field. This is indicated in Fig. 4. While each homodyne detector separately will record excess noise in accordance with Fig. 3, the noise of the two detectors can be strongly correlated or anticorrelated to a degree higher than possible with classical states of light.

The natural quadratures for expressing the two-point correlations are

$$
\hat{P}_{\mathrm{j}}^{ \pm}(\theta, \Omega, \mathbf{k})=\hat{P}_{\mathrm{j}}(\theta, \Omega, \mathbf{k}) \pm g \hat{P}_{\mathrm{j}}(\theta, \Omega,-\mathbf{k}),
$$


$j=1,2$ that measure the sum and difference of a fixed quadrature angle $\theta$ at two opposite points on the emission ring. Furthermore, we have allowed for the attenuation of one of the beams through the parameter $0 \leqslant g \leqslant 1$. This is a well-known method of optimizing nonperfect EPR correlations [4]. The corresponding spectrum is given by

$$
\begin{aligned}
S_{\mathrm{j}}^{ \pm}(\theta, \Omega, \mathbf{k}) & =\frac{\left\langle\hat{P}_{\mathrm{j}, \text { out }}^{ \pm}(\theta, \Omega, \mathbf{k}) \hat{P}_{\mathrm{j}, \text { out }}^{ \pm}(\theta,-\Omega, \mathbf{k})\right\rangle}{\left\langle\hat{P}_{\mathrm{j}, \text { in }}^{ \pm}(\theta, \Omega, \mathbf{k}) \hat{P}_{\mathrm{j}, \text { in }}^{ \pm}(\theta,-\Omega, \mathbf{k})\right\rangle} \\
& =\frac{1+g^{2}}{2} S_{\mathrm{j}}(\theta, \Omega, \mathbf{k}) \pm g S_{\mathrm{j}}^{\mathrm{CC}}(\theta, \Omega, \mathbf{k}) .
\end{aligned}
$$

Here we have limited to the case where the same quadrature amplitude $(\theta)$ is measured in the two different spatial points. This turns out to be sufficiently general since the case with two independent quadrature phases $\theta_{1}$ and $\theta_{2}$ can be obtained after the transformation $2 \theta \rightarrow \theta_{1}+\theta_{2}$. Thus all parameter regions can be accessed by adjusting only $\theta$. The spectrum $S_{\mathrm{j}}(\theta, \Omega, \mathbf{k})$ was introduced already in Sec. IV, while the cross correlation spectrum is defined as

$$
S_{\mathrm{j}}^{\mathrm{CC}}(\theta, \Omega, \mathbf{k})=\frac{\left\langle\hat{P}_{\mathrm{j}, \text { out }}(\theta, \Omega, \mathbf{k}) \hat{P}_{\mathrm{j}, \text { out }}(\theta,-\Omega,-\mathbf{k})\right\rangle}{\left\langle\hat{P}_{\mathrm{j}, \text { in }}(\theta, \Omega, \mathbf{k}) \hat{P}_{\mathrm{j}, \mathrm{in}}(\theta,-\Omega, \mathbf{k})\right\rangle},
$$

and we have made use of the symmetry under the transformation $\mathbf{k} \rightarrow-\mathbf{k}$. The cross correlation spectrum can be evaluated using Eqs. (12). It follows that

$$
\begin{aligned}
S_{\mathrm{j}}^{ \pm}(\theta, \Omega, \mathbf{k})= & \frac{1+g^{2}}{2} u_{\mathrm{j}}(\Omega, \mathbf{k}) \pm g\left[v_{\mathrm{j}}(\Omega, \mathbf{k}) e^{-2 i \theta}\right. \\
& \left.+v_{\mathrm{j}}^{*}(\Omega, \mathbf{k}) e^{2 i \theta}\right],
\end{aligned}
$$

which is valid only for $\mathbf{k} \neq 0$. The functions $u_{j}$ and $v_{j}$ were defined in Eqs. (18). It is observed immediately that

$$
S_{\mathrm{j}}^{-}(\theta+\pi / 2, \Omega, \mathbf{k})=S_{\mathrm{j}}^{+}(\theta, \Omega, \mathbf{k}),
$$

which will be shown to simplify the EPR criteria stated below.

The spectra $S_{j}^{ \pm}$can be used to express the EPR criteria formulated by Reid and Drummond [13]. EPR correlations exist in regions determined by the inequality

$$
S_{\mathrm{j}}^{+}(\theta, \Omega, \mathbf{k}) S_{\mathrm{j}}^{-}(\theta+\pi / 2, \Omega, \mathbf{k})<\frac{1}{4} .
$$

The seeming paradox, as encountered by Einstein, Podolsky, and Rosen, is that this allows $\hat{P}(\theta, \Omega, \mathbf{k})+\hat{P}(\theta, \Omega,-\mathbf{k})$ and $\hat{P}(\theta+\pi / 2, \Omega, \mathbf{k})-\hat{P}(\theta+\pi / 2, \Omega,-\mathbf{k})$ to be measured simultaneously with, in principle, arbitrary precision limited only by the strength of the correlations. Due to the degeneracy expressed by Eq. (25), the EPR criteria in this case simply reduce to

$$
S_{\mathrm{j}}^{+}(\theta, \Omega, \mathbf{k})<\frac{1}{2}
$$
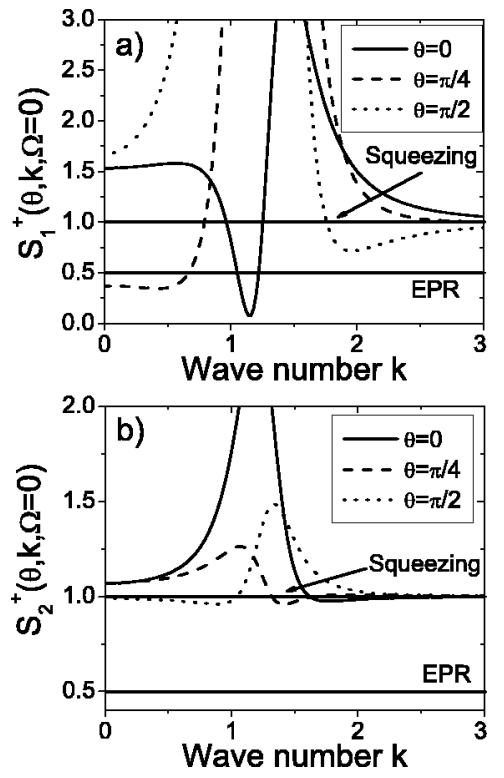

FIG. 5. Two-point correlation functions for the fundamental field (a) and second harmonic field (b) with $\Delta_{1}=0, \xi=10,\left|A_{1}\right|^{2}$ $=4.0, g=1$, and three values of the quadrature angle $\theta$. The instability threshold is $\left|A_{1}\right|^{2}=5.58$ with a critical wave number $k_{\mathrm{c}}$ $=1.46$. Two-mode squeezing is found when the correlation function is below unity, and the EPR criterion requires reduction below $1 / 2$.

The degeneracy also ensures that the entanglement criteria of the two-mode quantum states, as was recently formulated by Duan et al. [37], coincide with the above EPR criteria.

The upper bound of 1/2 for the EPR criterion in Eq. (27) poses a stronger correlation requirement than squeezing, where the upper bound is 1 . Hence the EPR criterion demands reduction of the noise in the sum or difference of two separated beams below the vacuum noise level of a single beam. In contrast, squeezing in the $\hat{P}^{ \pm}$quadratures is merely reduction below the vacuum noise level of both beams differing a factor of 2 from the single beam vacuum noise level.

Both two-mode squeezing and EPR correlations are found in the system for a wide range of parameters where the spatial instability exists. In general, the strongest nonclassical correlations appear close to the critical wave vector $k_{\mathrm{c}}$ characterizing the spatial instability. Representative examples are displayed in Figs. 5(a) and 5(b) for the fundamental and second harmonic fields, respectively, and plotted for several different values of the quadrature angle $\theta$. The nonclassical correlations are strongest in the fundamental field where the EPR criterion can be met. Here the noise is reduced to 0.07 (for $\theta=0$ ) which is far below the EPR limit of 0.5. The noise reduction in the second harmonic field is more modest, typically to a level around 0.90 . This is below the vacuum noise level for two beams, and hence a signature of twomode squeezing. However, the second harmonic correlations appear to be not strong enough to cross the EPR boundary.

Nonperfect correlations can be optimized by attenuating one of the spatially separated beams. This was the reason for introducing the attenuation parameter $g$ in Eq. (21). Since the correlation between $\hat{P}_{j}(\mathbf{k})$ and $\hat{P}_{j}(-\mathbf{k})$ is not perfect, it is 


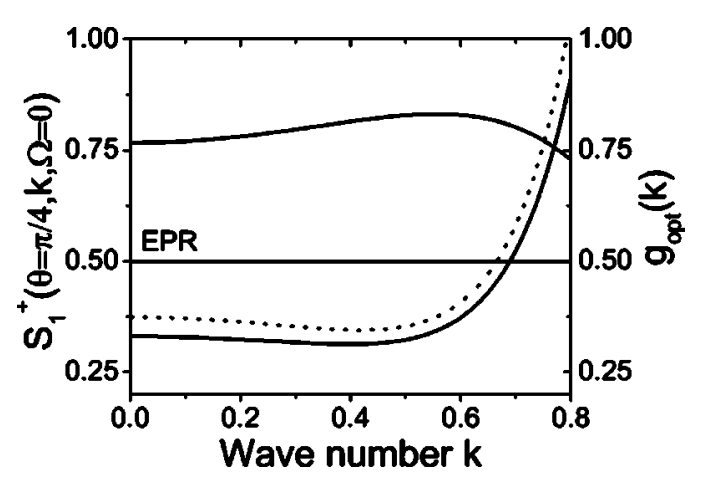

FIG. 6. Optimization of EPR correlations of the fundamental field by varying the attenuation parameter. The dotted curve shows the correlation for $g=1$ while the solid curve is with $g=g_{\text {opt }}(k)$. The optimum attenuation parameter depends on the wave number $k$ and is also displayed in the plot. All other parameters are the same as in Fig. 5.

favorable to infer $\hat{P}_{j}(\mathbf{k})+g \hat{P}_{j}(-\mathbf{k})$, given the value of the attenuation parameter $g$ is known. An optimum exists for each fixed set of parameters, allowing to maximize the correlations in each separate far-field point. For the strongest EPR correlations in Fig. 5 ( $\theta=0, k=1.15), g=1$ turns out to be optimum. For other parameters this is not the case. From Eq. (22) it is straightforward to see that the optimum attenuation parameter for minimizing $S_{1}^{+}(\theta, \Omega, \mathbf{k})$ is given by

$$
g_{\text {opt }}(\theta, \Omega, \mathbf{k})=-\frac{S_{\mathrm{j}}^{\mathrm{CC}}(\theta, \Omega, \mathbf{k})}{S_{\mathrm{j}}(\theta, \Omega, \mathbf{k})} .
$$

An example of this optimization is shown in Fig. 6 for the fundamental field. For fixed frequency and quadrature angle, the EPR correlations are increased significantly by attenuating one beam according to $g_{\text {opt }}$.

Attempts to increase the correlations of the second harmonic field beyond the EPR limit by changing the attenuation parameter were not successful despite a substantial search in the very large parameter space present in the system. This suggests the conclusion that while strong EPR correlations are readily present in the fundamental field the nonclassical correlations in the second harmonic field are limited to squeezing. Nonetheless, the simultaneous existence of nonclassical correlations in both the fundamental and the second harmonic fields is a surprising finding that is unique for SHG. This has no counterpart in an ordinary OPO and appears as a consequence of the spatial instability that creates off-axis emitted beams in both the fundamental and the second harmonic fields. Even in the spatially extended OPO, where a spatial instability does exist, spatial quantum correlations have only been predicted in the fundamental field and not in the pump [20]. The reason for this pronounced difference between SHG and the OPO can be traced back to the existence of an oscillation threshold in the latter. Below this threshold, the equation for the pump field decouples such that no spatial dynamics is created in this field. In SHG no oscillation threshold exists and spatial modulation is created in both fields.

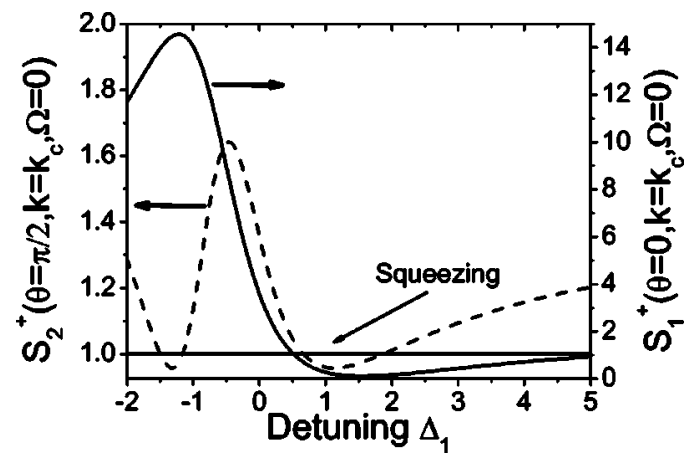

FIG. 7. Two-point correlation function for the fundamental field (solid curve) and second harmonic field (dashed curve) for $\left|A_{1}\right|^{2}$ $=4.0, \xi=10, g=1$, and at the critical wave number $k=k_{\mathrm{c}}$. The spatial instability threshold is $\left|A_{1}\right|^{2}=5.58$.

An important tuning parameter is the cavity detuning $\Delta_{1}$. Figure 7 gives an example of the variation of the two-point correlation function with detuning for $\xi=10$. For these parameters the spatial instability exists for $\Delta_{1} \geqslant-2$ [23], and in this region a pronounced spatial modulation of the twopoint correlation functions is observed. The significant variations of the correlation functions with detuning allow tuning to regions with either excess noise or strong nonclassical correlations.

Interesting time dependent behavior of the two-point correlation functions is found when varying the cavity detuning. A representative example is given in Fig. 8. For large positive values of the detuning the best correlations are observed at zero frequency. Decreasing the detuning translates the position of the optimum correlations to a nonzero frequency. As noted previously, such a behavior would be lucrative in an experimental situation since this would make the measurements less sensitive to technical noise.

\section{CONCLUSIONS}

In the current paper the spatial structure of quantum correlations in singly resonant second-harmonic generation was investigated. By quantizing the cavity mean-field equation for the fundamental field, the spatial distribution of quantum

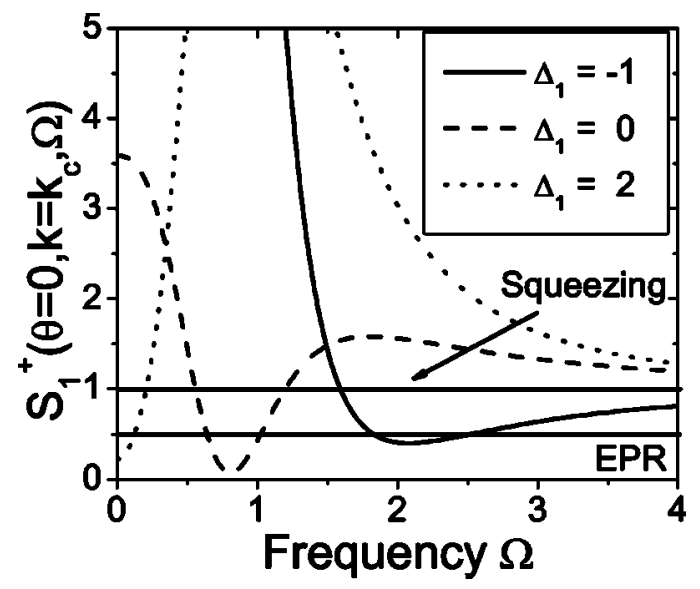

FIG. 8. Plot of the fundamental two-point correlation function as a function of frequency with $\xi=10,\left|A_{1}\right|^{2}=4.0, g=1, k=k_{\mathrm{c}}$, and three different values of the detuning $\Delta_{1}$. 
noise of both the fundamental and second-harmonic output fields could be computed. A surprising result was the demonstration of EPR correlations in second-harmonic generation which are otherwise known to occur in the opposite process of down-conversion in optical parametric oscillators. The EPR correlations are caused by off-axis emission creating strongly correlated beams due to the requirement of momentum conservation in the transverse plane. A notable difference compared to the OPO is that also the secondharmonic possess nonclassical spatial correlations in SHG. Strong squeezing was also identified in the system in farfield points where $\mathbf{k}=0$. Hence, this allows for the intriguing situation of having both squeezing and EPR correlations generated simultaneously in the spatial structure of the emitted light. This emphasizes the amazing variety of spatial dynam- ics present in singly resonant SHG as evident here in the quantum noise that constitutes the predecessor for the equally rich pattern formation dynamics happening above instability threshold.

\section{ACKNOWLEDGMENTS}

I would like to acknowledge Mark Saffman for valuable discussions and Willem Vos for useful comments on the manuscript. This work is part of the research program of the "Stichting voor Fundamenteel Onderzoek der Materie (FOM)," which was financially supported by the "Nederlandse Organisatie voor Wetenschappelijk Onderzoek (NWO)."
[1] A. Einstein, B. Podolsky, and N. Rosen, Phys. Rev. 47, 777 (1935).

[2] A. Aspect, J. Dalibard, and G. Roger, Phys. Rev. Lett. 49, 1804 (1982).

[3] J.S. Bell, Physics 1, 195 (1965).

[4] Z.Y. Ou, S.F. Pereira, H.J. Kimble, and K.C. Peng, Phys. Rev. Lett. 68, 3663 (1992).

[5] A. Kuzmich, I.A. Walmsley, and L. Mandel, Phys. Rev. Lett. 85, 1349 (2000).

[6] A. Furusawa, J.L. Sørensen, S.L. Braunstein, C.A. Fuchs, H.J. Kimble, and E.S. Polzik, Science 282, 706 (1998).

[7] T. Zhang, K.W. Goh, C.-W. Chou, P. Lodahl, and H.J. Kimble, Phys. Rev. A 67, 033802 (2003).

[8] X.Y. Li, Q. Pan, J.T. Jing, J. Zhang, C.D. Xie, and K.C. Peng, Phys. Rev. Lett. 88, 047904 (2002).

[9] M. Hillery, Phys. Rev. A 61, 022309 (2000).

[10] E.S. Polzik, J. Carri, and H.J. Kimble, Phys. Rev. Lett. 68, 3020 (1992).

[11] L.A. Wu, H.J. Kimble, J.L. Hall, and H. Wu, Phys. Rev. Lett. 57, 2520 (1986).

[12] P. Grangier, R.E. Slusher, A. LaPorta, B. Yurke, and M.J. Potasek, Phys. Rev. Lett. 59, 2566 (1987).

[13] M. Reid and P.D. Drummond, Phys. Rev. Lett. 60, 2731 (1988); P.D. Drummond and M. Reid, Phys. Rev. A 41, 3930 (1990)

[14] G.-L. Oppo, M. Brambilla, and L.A. Lugiato, Phys. Rev. A 49, 2028 (1994).

[15] S. Longhi, Phys. Rev. A 53, 4488 (1996).

[16] G.J. Valcárcel, K. Staliunas, E. Roldán, and V.J. SánchezMorcillo, Phys. Rev. A 54, 1609 (1996).

[17] L.A. Lugiato and A. Gatti, Phys. Rev. Lett. 70, 3868 (1993).

[18] M.I. Kolobov, Rev. Mod. Phys. 71, 1539 (1999).
[19] A. Gatti, H. Wiedemann, L.A. Lugiato, I. Marzoli, G.-L. Oppo, and S.M. Barnett, Phys. Rev. A 56, 877 (1997).

[20] A. Gatti, L.A. Lugiato, K.I. Petsas, and I. Marzoli, Europhys. Lett. 46, 461 (1999).

[21] C. Etrich, U. Peschel, and F. Lederer, Phys.Rev. E 56, 4803 (1997).

[22] P. Lodahl and M. Saffman, Phys. Rev. A 60, 3251 (1999).

[23] P. Lodahl and M. Saffman, Opt. Commun. 184, 493 (2000).

[24] P. Lodahl and M. Saffman, Opt. Lett. 27, 110 (2002); Opt. Lett. 27, 551(E) (2002).

[25] M. Bache, P. Scotto, R. Zambrini, M. San Miguel, and M. Saffman, Phys. Rev. A 66, 013809 (2002).

[26] M. Bache, P. Lodahl, A.V. Mamaev, M. Marcus, and M. Saffman, Phys. Rev. A 65, 033811 (2002).

[27] M. Vaupel, A. Maitre, and C. Fabre, Phys. Rev. Lett. 83, 5278 (1999).

[28] A.V. Mamaev, P. Lodahl, and M. Saffman, Opt. Lett. 28, 31 (2003).

[29] S.F. Pereira, M. Xiao, H.J. Kimble, and J.L. Hall, Phys. Rev. A 38, 4931 (1988).

[30] M.J. Collett and R.B. Levien, Phys. Rev. A 43, 5068 (1991).

[31] R. Paschotta, M. Collett, P. Kürz, K. Fiedler, H.-A. Bachor, and J. Mlynek, Phys. Rev. Lett. 72, 3807 (1994).

[32] C.W. Gardiner and M.J. Collett, Phys. Rev. A 31, 3761 (1985).

[33] A. Gatti and L. Lugiato, Phys. Rev. A 52, 1675 (1995).

[34] C. Fabre and S. Reynaud, in Fundamental Systems in Quantum Optics, edited by J. Dalibard, J.M. Raimond, and J. Zinn-Justin (Elsevier, Amsterdam, 1992).

[35] M.O. Scully and M.S. Zubairy, Quantum Optics (Cambridge University Press, Cambridge, 1997).

[36] H.P. Yuen and V.W.S. Chan, Opt. Lett. 8, 177 (1983).

[37] L.-M. Duan, G. Giedke, J.I. Cirac, and P. Zoller, Phys. Rev. Lett. 84, 2722 (2000). 